\title{
Screening key miRNAs for human hepatocellular carcinoma based on miRNA-mRNA functional synergistic network
}

\author{
C. J. ZHANG ${ }^{1, *}$, H. J. DU² \\ ${ }^{1}$ Department of Tumor, Linyi People' Hospital of Shandong Province; ${ }^{2}$ Department of Tumor, Feixian People' Hospital of Shandong Province
}

${ }^{*}$ Correspondence: zhangchj1002@163.com

Received November 29, 2016 / Accepted March 8, 2017

\begin{abstract}
The safety of miRNAs has been proven and the prophylactic use of miRNA-based approaches may be foreseen for patients with hepatocellular carcinoma (HCC). However, the underlying regulatory mechanism of miRNAs in HCC has not been fully clarified. Using bioinformatic analyses, we compared data of miRNA and mRNA expression profiling of HCC from Gene Expression Omnibus (GEO) database, respectively. Differentially expressed miRNAs and differentially expressed genes (DEGs) were identified. Based on the miRTarBase predictions, the miRNA-dependent regulatory network was constructed. In total, comparative analysis of five mRNA datasets and two miRNA datasets led to 1449 DEGs and 17 differentially expressed miRNAs, respectively. Based on the predictions, a global miRNA-mRNA regulatory network was then constructed, which encompassed 451 miRNA target gene pairs whose expressions were inversely correlated. Three miRNAs (miR-641, miR-507 and miR-501-5p) were the most connected miRNAs that regulated a large number of genes, among which miR-641 and miR-507 were novel miRNAs altered in HCC. We suggested that miR-501-5p will represent a powerful therapeutic target for HCC. Moreover, four up-regulated miRNAs (miR-769-3p, miR-941, miR-362-3p and miR16-1) and one down-regulated miRNA (miR-581) may be involved in HCC. Additionally, two targets of MAPK8 and SRPK2 were also detected in this study, whose roles in HCC will be notable. In conclusion, we developed an integrative approach to construct an interactive global network of miRNA-mRNA, which can contribute to refine miRNA target predictions for developing new therapeutic approaches.
\end{abstract}

Key words: Hepatocellular carcinoma, miRNAs, regulatory network, integrated analysis

Hepatocellular carcinoma (HCC) is the primary malignancy of the liver and it is a leading unnatural death worldwide [1]. Even so, HCC still has a poor prognosis owing to largely ineffective therapeutic options currently. MicroRNAs (miRNAs) have emerged as potential therapeutic molecules and targets according to the fact that they are important in tumorigenesis of HCC by regulating the expression of protein-coding genes posttranscriptionally $[2,3]$.

Recent advancements shed light on the molecular mechanisms of HCC tumorigenesis. The results from next-generation sequencing revealed that several miRNAs are deregulated in HCC compared with matched nonneoplastic tissue $[4,5]$. There are several signaling pathways involved in the HCC pathogenesis, including the p53, PI3K/Akt/mTOR, Ras/ Raf/MEK/ERK, IGFR, and Wnt/b-catenin pathways, which are regulated by a set of miRNAs [6-9]. Various aberrantly expressed miRNAs play important roles in the cell-cycle progression of HCC, including miR-34 [10], miR-26 [11],
miR-124 [12], miR-203 [12], miR-195, miR-221[13], miR-335 [14]. Moreover, the aberrant expression of various miRNAs can affect the functionality of apoptosis pathways, such as miR483 [15], miR-122 [16], let-7 [17], miR-21 [18], miR-193 [19] and miR-101 [20]. In addition, the role of miRNAs in HCC has been established with molecular studies and the animal models of HCC, which confirmed the importance of miRNA deregulation in HCC [21].

Actually, ample evidence suggests that the development of miRNA-based therapeutics could be a clinically viable approach for patients with HCC. It is well known that miRNAs appear to have an essential role in the modulation of complex cross-talk that exists between pathways affecting HCC development and progression. To investigate this issue, we here performed a comprehensive analysis by integrating of the miRNA and mRNA expression profiles of HCC. Then, differentially expressed miRNA-target genes were predicted, and a miRNA-target regulatory network was constructed based 
on them. The global miRNA-mRNA functional synergistic network may further clarify the roles of miRNAs in HCC and facilitate the development of novel, targeted therapeutic strategies against HCC.

\section{Patients and methods}

Microarray datasets of HCC. To obtain maximal information regarding the difference of mRNA and miRNA expression profiling between HCC and normal tissues, we searched the Gene Expression Omnibus database (GEO, http://www.ncbi.nlm.nih.gov/geo) [22] with the following keywords: "carcinoma, hepatocellular" [MeSH Terms] OR hepatoma [All Fields] AND "Homo sapiens" [porgn] AND "Expression profiling by array" [Filter]. We only selected the original experimental microarray studies that analyzed mRNA and miRNA expression profiling between HCC and normal tissues in the human.

Identification of differentially expressed miRNAs and mRNAs. The raw data of microarray datasets was preprocessed via background correction and normalization. A gene specific t-test was carried out between HCC and normal tissues and p-value and effect size of each microarray study was calculated. P-value from multiple studies was combined using Fisher test, and the random effects model was used to combine effect size from multiple studies. Additionally, we also calculated the fold change of all differentially expressed miRNAs according to the method of previous article [23]. We set FDR $<0.01$ as the cutoff of differentially expressed miRNAs (Suppl Table S1). Moreover, we selected genes with FDR $<0.001$ as differentially expressed mRNAs.

Recognition of targets for differently expressed miRNA. We downloaded the target genes for human miRNAs from the database of miRTarBase (http://mirtarbase.mbc.nctu.edu. tw/). Targets of differentially expressed miRNAs in HCC were predicted. Moreover, we selected the differentially expressed target genes whose expression was inversely correlated with that of miRNAs for network construction. The miRNA-target genes interaction network was visualized using Cytoscape (available at http://www.cytoscape.org/) [23].
Functional annotation of DEGs. Online based software GeneCodis3 was used for functional analysis of DEGs [24]. To uncover the biological functions of the DEGs, Gene Ontology (GO) enrichment analysis was conducted [25]. Additionally, biological pathway enrichment analysis was also performed based on the Kyoto Encyclopedia of Genes and Genomes (KEGG) database [26].

Patients and tissues. Five pairs of HCC tumor samples and the healthy tissue adjacent to carcinoma were obtained at the time of surgery, and were immediately frozen in liquid nitrogen. The written informed consent forms were obtained from patients or legal guardians of the patients. All protocols were approved by the Medical Ethics Committee of our hospital.

QRT-PCR validation. The total RNA from each sample was extracted using the Trizol method (Takara) following the manufacturer's protocols. For each replicate, cDNA was synthesized from $1 \mu \mathrm{g}$ RNA using Superscript Reverse Transcriptase II (TOYOBO). The qRT-PCR reaction was carried out in BIO-RAD IQ5 real-time PCR system with Power SYBR Green PCR Master Mix (Applied Biosystems/Life Technologies, Carlsbad, CA). Relative gene expression was analyzed using $2^{-\Delta \Delta C t}$ method. The human GAPDH gene was used as endogenous controls for RNA load and gene expression in the analysis.

\section{Results}

DEGs in the integrated analysis of microarray datasets. Totally, 5 datasets of mRNA expression profiling were obtained according to the inclusion criteria, and the GEO IDs were GSE54236, GSE33006, GSE25097, GSE14520 and GSE14323. Moreover, 2 datasets of miRNA expression profiling (GSE41874 and GSE36915) were enrolled in. We subsequently performed the integrated analysis of mRNA expression profiling which contained $639 \mathrm{HCC}$ and 644 normal tissues samples, while the integrated analysis of miRNA expression profiling contained $74 \mathrm{HCC}$ and 25 normal tissues samples. Individual studies for integrated analysis were displayed in Table 1 . The integrated analysis showed that a total of 17 miRNAs were found to be consistently differentially

Table 1. Characteristics of the eligible mRNA and miRNA expression profiling of HCC

\begin{tabular}{|c|c|c|c|c|}
\hline GEO ID & Author & Platform & Samples (N:P) & Year \\
\hline \multicolumn{5}{|c|}{ mRNA expression profiling } \\
\hline GSE54236 & Villa E & GPL6480 Agilent-014850 Whole Human Genome Microarray 4x44K G4112F (Probe Name version) & $80: 81$ & 2014 \\
\hline GSE33006 & Huang Y & GPL570 [HG-U133_Plus_2] Affymetrix Human Genome U133 Plus 2.0 Array & $3: 3$ & 2012 \\
\hline GSE25097 & Tung EK & GPL10687 Rosetta/Merck Human RSTA Affymetrix 1.0 microarray, Custom CDF & 303:268 & 2011 \\
\hline GSE14520 & Cui J Roessler S & $\begin{array}{l}\text { GPL571 [HG-U133A_2] Affymetrix Human Genome U133A } 2.0 \text { Array/GPL3921 [HT_HG- } \\
\text { U133A] Affymetrix HT Human Genome U133A Arr }\end{array}$ & 239:249 & 2010 \\
\hline GSE14323 & Mas VR & GPL571 [HG-U133A_2] Affymetrix Human Genome U133A 2.0 Array & 19.38 & 2009 \\
\hline \multicolumn{5}{|c|}{ miRNA expression profiling } \\
\hline GSE41874 & Morita K & miRCURY LNA microRNA Array, v.10.0 - hsa, mmu \& rno & $4: 6$ & 2012 \\
\hline GSE36915 & Shih TC & Illumina Human v2 MicroRNA expression beadchip & $21: 68$ & 2012 \\
\hline
\end{tabular}




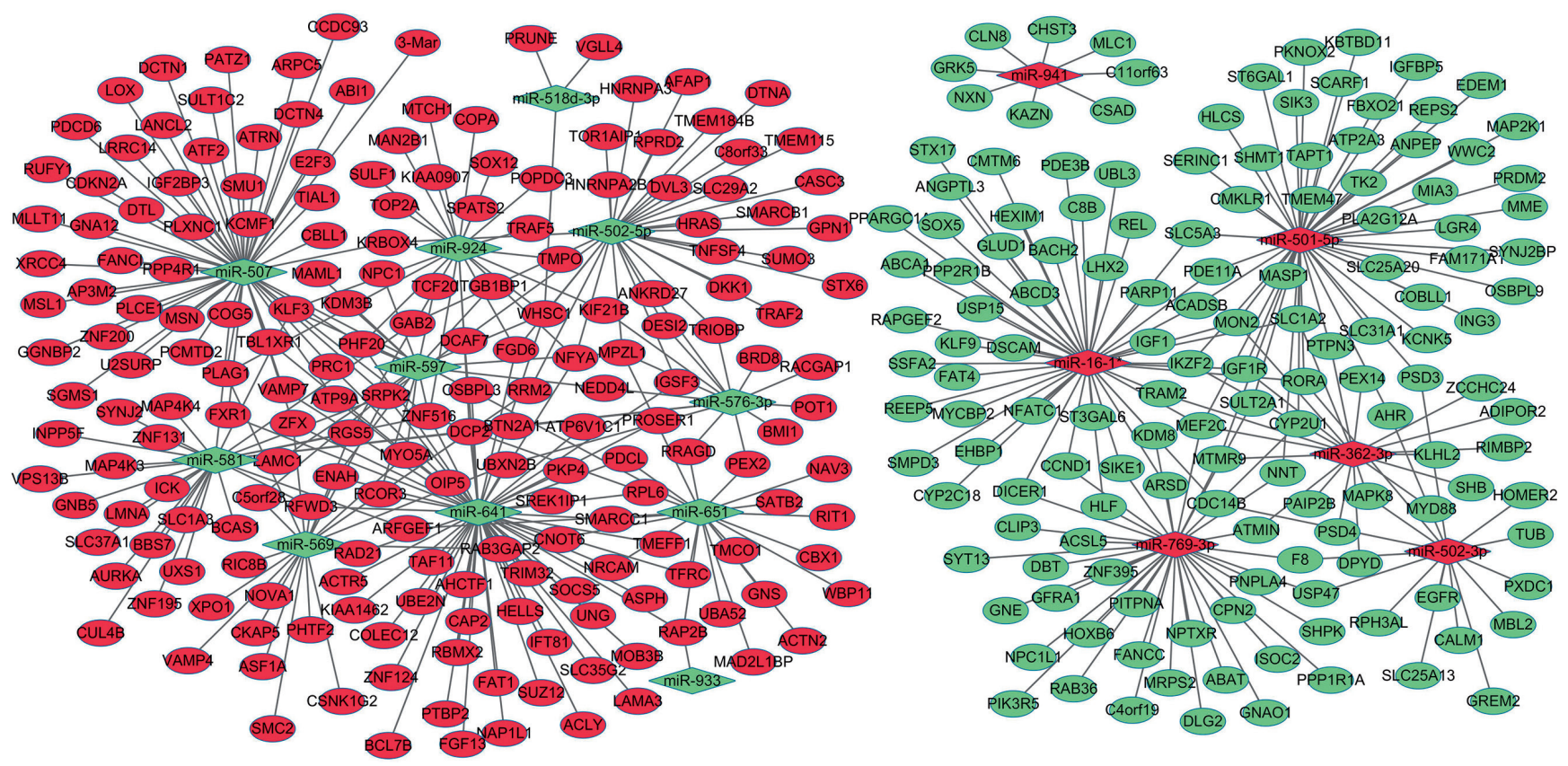

Figure 1. The posttranscriptional regulatory network of miRNAs and target genes in HCC. Diamonds and ellipses represent the miRNAs and targets, respectively. The red and green colors represent up-regulation and down-regulation, respectively.

expressed with FDR $<0.01$, including 6 miRNAs up-regulated and 11 miRNAs down-regulated in HCC (Table 2). Furthermore, integrated analysis led to 1449 DEGs with $\mathrm{FDR}<0.001$, including 784 up-regulated and 665 down-regulated DEGs in HCC.

Regulatory network of miRNAs and target genes in HCC. According to the database of miRTarBase, we predicted the targets of the differentially expressed miRNAs in HCC. Totally, we obtained $451 \mathrm{miRNA}$-target gene pairs whose expressions were inversely correlated, including 180 down-regulated targets and 271 up-regulated targets. Moreover, a global miRNA-target gene regulatory network was constructed based on the miRNA-target gene pairs (Figure 1). In the network, the top three miRNAs regulating the most target genes were hsa-miR-641, hsa-miR-507 and hsa-miR-501-5p.

Functional annotation of differentially expressed target genes. GO enrichment analysis of differentially expressed target genes was performed to understand their biological functions. In our analysis, 3 categories were detected respectively using web-based software GENECODIS. We found that the significantly enriched GO term for molecular functions was protein binding (GO: 0005515, FDR=1.32E-31), while the GO term for biological processes was regulation of transcription (GO: 0006355, FDR=2.86E-06), toll signaling pathway (GO:0008063, FDR=1.18E-03), toll-like receptor 1 signaling pathway (GO:0034130, $\mathrm{FDR}=1.03 \mathrm{E}-03)$, toll-like

Table 2. List of differentially expressed miRNAs in HCC

\begin{tabular}{|c|c|c|c|c|c|c|c|}
\hline miRNAs & P-Value & FDR & Fold change & miRNAs & P-Value & FDR & Fold change \\
\hline \multicolumn{8}{|c|}{ Up-regulated miRNAs } \\
\hline hsa-miR-501-5p & 2.53E-08 & 3.59E-06 & 1.479 & hsa-miR-362-3p & $2.17 \mathrm{E}-06$ & 7.71E-05 & 1.467 \\
\hline hsa-miR-769-3p & $1.10 \mathrm{E}-07$ & $1.04 \mathrm{E}-05$ & 1.322 & hsa-miR-502-3p & $5.20 \mathrm{E}-04$ & $9.75 \mathrm{E}-03$ & 1.311 \\
\hline hsa-miR-941 & $1.77 \mathrm{E}-06$ & 7.19E-05 & 1.425 & hsa-miR-16-1* & $5.70 \mathrm{E}-04$ & $9.75 \mathrm{E}-03$ & 1.314 \\
\hline \multicolumn{8}{|c|}{ Down-regulated miRNAs } \\
\hline hsa-miR-933 & $1.54 \mathrm{E}-08$ & $3.59 \mathrm{E}-06$ & 0.764 & hsa-miR-581 & $2.35 \mathrm{E}-04$ & $6.06 \mathrm{E}-03$ & 0.818 \\
\hline hsa-miR-924 & 5.39E-07 & $3.06 \mathrm{E}-05$ & 0.739 & hsa-miR-576-3p & $3.66 \mathrm{E}-04$ & $8.67 \mathrm{E}-03$ & 0.840 \\
\hline hsa-miR-651 & $5.29 \mathrm{E}-07$ & $3.06 \mathrm{E}-05$ & 0.730 & hsa-miR-507 & 4.36E-04 & $9.52 \mathrm{E}-03$ & 0.797 \\
\hline hsa-miR-502-5p & $7.45 \mathrm{E}-07$ & $3.53 \mathrm{E}-05$ & 0.784 & hsa-miR-569 & $5.80 \mathrm{E}-04$ & $9.75 \mathrm{E}-03$ & 0.914 \\
\hline hsa-miR-641 & $3.22 \mathrm{E}-06$ & $1.02 \mathrm{E}-04$ & 0.867 & hsa-miR-518d-3p & $5.84 \mathrm{E}-04$ & $9.75 \mathrm{E}-03$ & 0.880 \\
\hline hsa-miR-597 & 4.54E-05 & $1.29 \mathrm{E}-03$ & 0.882 & & & & \\
\hline
\end{tabular}




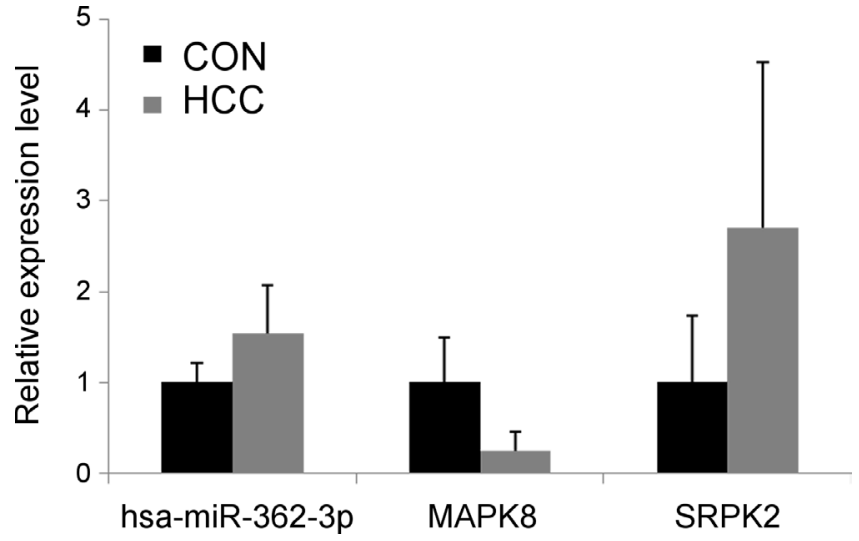

Figure 2. Validation of microarray-based detection of differentially expressed miRNAs and mRNAs in the HCC tissues by qRT-PCR. ${ }^{*} \mathrm{P}<0.01$.

receptor 2 signaling pathway (GO:0034134, $\mathrm{FDR}=1.03 \mathrm{E}-03)$ and toll-like receptor 4 signaling pathway (GO:0034142, $\mathrm{FDR}=1.21 \mathrm{E}-03)$. Moreover, significantly enriched GO term for cellular component was cytoplasm (GO: 0005737, $\mathrm{FDR}=7.52 \mathrm{E}-25$ ). (Table 3 ) The pathway enrichment analysis was also conducted to further evaluate the biological pathways the DEGs involved. The most significantly enriched pathway was Glioma $(\mathrm{FDR}=2.80 \mathrm{E}-08)$. Furthermore, pathway in cancer $(\mathrm{FDR}=2.75 \mathrm{E}-06)$ was also found to be significantly enriched. (Table 4)

QRT-PCR validation. The tumor and the normal tissue adjacent to carcinoma from patients with HCC were used to verify the expression of miRNAs and their target genes by integrated analysis. The miR-362-3p, MAPK8 and SRPK2 were selected for qRT-PCR validation. The qRT-PCR results showed that the expression pattern of selected genes in HCC and normal tissues was nearly consistent with that in the integrated analysis. As displayed in Figure 2, the expression of miR-362-3p and SRPK2 were higher in HCC than in normal tissues, while the expression of MAPK8 was dramatically lower in HCC when compared with the normal tissues. Additionally, we performed electronic validation of several identified miRNAs (miR-362-3p, miR-501-5p, miR-502-3p, miR-576-3p, miR-641, miR-651 and miR-769-3p) in the TCGA dataset (Figure 3 ). The result was consistent with the integrated analysis, which suggested that our result was believable.

\section{Discussion}

Accumulating evidences suggested that the deregulation of miRNAs could modulate tumor cell proliferation, invasion and metastasis during the progression of various human cancers [27]. Obviously, the change in the endogenous expression of miRNAs is a crucial mechanism in the hepatocarcinogenesis. Considering that miRNAs and their target mRNAs function cooperatively, we sought to scrutinize the effects of miRNAs by integrated analysis. Totally, five mRNA
Table 3. Top 15 enriched GO terms of differentially expressed target genes

\begin{tabular}{|c|c|c|c|}
\hline GO ID & GO Term & Count & FDR \\
\hline \multicolumn{4}{|c|}{ Biological process } \\
\hline GO:0006355 & $\begin{array}{l}\text { regulation of transcription, } \\
\text { DNA-dependent }\end{array}$ & 43 & $2.86 \mathrm{E}-06$ \\
\hline GO:0007165 & signal transduction & 31 & $2.57 \mathrm{E}-04$ \\
\hline GO:0045087 & innate immune response & 15 & $2.80 \mathrm{E}-04$ \\
\hline GO:0042493 & response to drug & 14 & $5.59 \mathrm{E}-04$ \\
\hline GO:0034130 & toll-like receptor 1 signaling pathway & 7 & $1.03 \mathrm{E}-03$ \\
\hline GO:0034134 & toll-like receptor 2 signaling pathway & 7 & $1.03 \mathrm{E}-03$ \\
\hline GO:0008063 & Toll signaling pathway & 7 & $1.18 \mathrm{E}-03$ \\
\hline GO:0007173 & $\begin{array}{l}\text { epidermal growth factor receptor } \\
\text { signaling pathway }\end{array}$ & 7 & $1.19 \mathrm{E}-03$ \\
\hline GO:0034142 & toll-like receptor 4 signaling pathway & 7 & $1.21 \mathrm{E}-03$ \\
\hline GO:0002224 & toll-like receptor signaling pathway & 7 & $1.24 \mathrm{E}-03$ \\
\hline GO:0002755 & $\begin{array}{l}\text { MyD88-dependent toll-like receptor } \\
\text { signaling pathway }\end{array}$ & 7 & $1.30 \mathrm{E}-03$ \\
\hline GO:0006357 & $\begin{array}{l}\text { regulation of transcription from RNA } \\
\text { polymerase II promoter }\end{array}$ & 11 & $1.70 \mathrm{E}-03$ \\
\hline GO:0006464 & protein modification process & 9 & $1.82 \mathrm{E}-03$ \\
\hline GO:0045444 & fat cell differentiation & 5 & $3.30 \mathrm{E}-03$ \\
\hline GO:0016574 & histone ubiquitination & 3 & $4.08 \mathrm{E}-03$ \\
\hline \multicolumn{4}{|c|}{ Cellular component } \\
\hline GO:0005737 & cytoplasm & 133 & $7.52 \mathrm{E}-25$ \\
\hline GO:0005634 & nucleus & 130 & $2.08 \mathrm{E}-22$ \\
\hline GO:0005829 & cytosol & 54 & $1.27 \mathrm{E}-08$ \\
\hline GO:0016020 & membrane & 76 & $9.45 \mathrm{E}-07$ \\
\hline GO:0005622 & intracellular & 46 & $1.80 \mathrm{E}-06$ \\
\hline GO:0005886 & plasma membrane & 68 & $2.33 \mathrm{E}-06$ \\
\hline GO:0005654 & nucleoplasm & 27 & $9.32 \mathrm{E}-06$ \\
\hline GO:0005794 & Golgi apparatus & 28 & $1.00 \mathrm{E}-05$ \\
\hline GO:0005730 & nucleolus & 35 & $3.12 \mathrm{E}-05$ \\
\hline GO:0005625 & soluble fraction & 15 & $2.13 \mathrm{E}-04$ \\
\hline GO:0009898 & internal side of plasma membrane & 5 & $6.15 \mathrm{E}-04$ \\
\hline GO:0031965 & nuclear membrane & 8 & $1.14 \mathrm{E}-03$ \\
\hline GO:0030175 & filopodium & 5 & $1.26 \mathrm{E}-03$ \\
\hline GO:0000785 & chromatin & 6 & $1.80 \mathrm{E}-03$ \\
\hline GO:0000228 & nuclear chromosome & 4 & $2.34 \mathrm{E}-03$ \\
\hline \multicolumn{4}{|c|}{ Molecular function } \\
\hline GO:0005515 & protein binding & 132 & $1.32 \mathrm{E}-31$ \\
\hline GO:0046872 & metal ion binding & 62 & $5.42 \mathrm{E}-07$ \\
\hline GO:0000166 & nucleotide binding & 48 & $6.88 \mathrm{E}-06$ \\
\hline GO:0003677 & DNA binding & 40 & $9.94 \mathrm{E}-05$ \\
\hline GO:0003682 & chromatin binding & 12 & $2.26 \mathrm{E}-04$ \\
\hline GO:0046982 & protein heterodimerization activity & 13 & $3.04 \mathrm{E}-04$ \\
\hline GO:0005524 & ATP binding & 33 & $6.86 \mathrm{E}-04$ \\
\hline GO:0004842 & ubiquitin-protein ligase activity & 11 & $1.25 \mathrm{E}-03$ \\
\hline GO:0005086 & $\begin{array}{l}\text { ARF guanyl-nucleotide exchange } \\
\text { factor activity }\end{array}$ & 4 & $1.34 \mathrm{E}-03$ \\
\hline GO:0046983 & protein dimerization activity & 7 & $1.45 \mathrm{E}-03$ \\
\hline GO:0008270 & zinc ion binding & 38 & $1.58 \mathrm{E}-03$ \\
\hline GO:0003700 & $\begin{array}{l}\text { sequence-specific DNA binding } \\
\text { transcription factor activity }\end{array}$ & 22 & $3.01 \mathrm{E}-03$ \\
\hline GO:0005314 & $\begin{array}{l}\text { high-affinity glutamate transmembrane } \\
\text { transporter activity }\end{array}$ & 2 & $3.21 \mathrm{E}-03$ \\
\hline GO:0003723 & RNA binding & 17 & 4.17E-03 \\
\hline GO:0005313 & $\begin{array}{l}\text { L-glutamate transmembrane transporter } \\
\text { activity }\end{array}$ & 3 & $4.19 \mathrm{E}-03$ \\
\hline
\end{tabular}




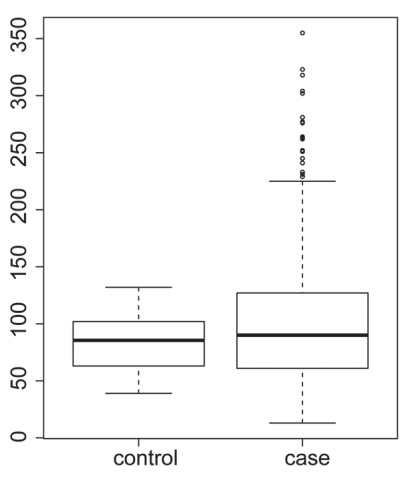

hsa-miR-769-3p|hsa-mir-769

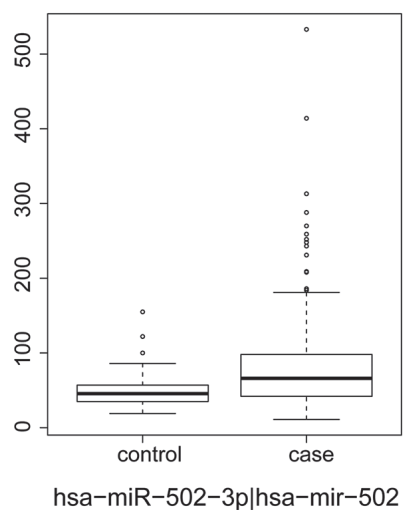

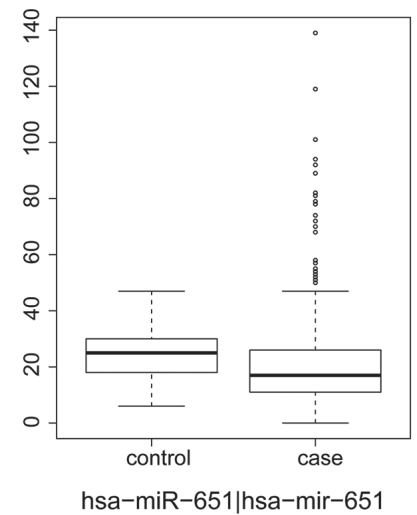
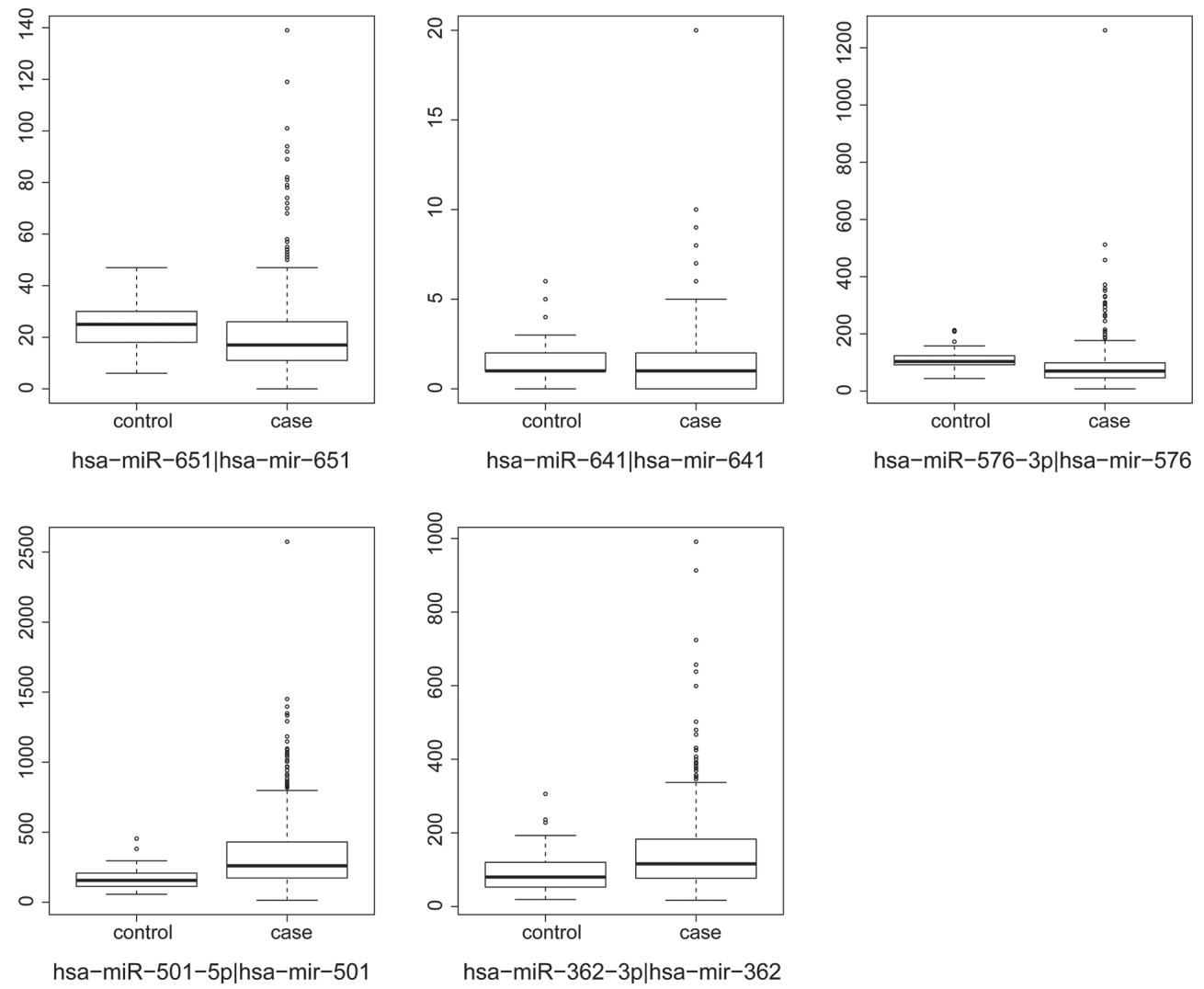

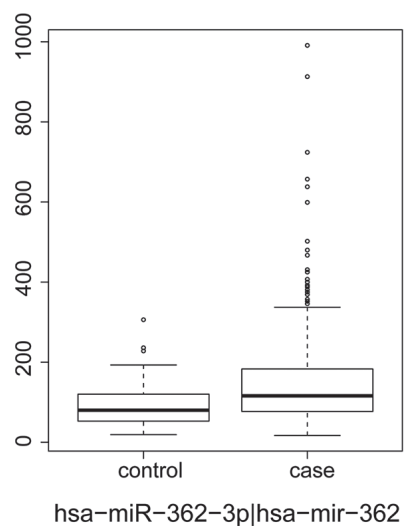

Figure 3. Electronic validation box plots of several miRNAs in the TCGA dataset.

expressions profiling and two miRNA expression profiling were obtained from GEO database. Integrated analysis led to a set of 17 differentially expressed miRNAs and 1449 DEGs in HCC. And 451 miRNA-target gene pairs were identified

Table 4. Top 15 ecriched KEGG pathways of differentially expressed target genes

\begin{tabular}{llcc}
\hline KEGG ID & KEGG term & Count & FDR \\
\hline hsa05214 & Glioma & 9 & $2.80 \mathrm{E}-08$ \\
hsa05218 & Melanoma & 10 & $8.77 \mathrm{E}-08$ \\
hsa05215 & Prostate cancer & 8 & $1.40 \mathrm{E}-07$ \\
hsa05223 & Non-small cell lung cancer & 7 & $5.28 \mathrm{E}-07$ \\
hsa05219 & Bladder cancer & 6 & $5.98 \mathrm{E}-07$ \\
hsa05200 & Pathways in cancer & 16 & $2.75 \mathrm{E}-06$ \\
hsa04730 & Long-term depression & 8 & $5.01 \mathrm{E}-06$ \\
hsa05213 & Endometrial cancer & 4 & $9.37 \mathrm{E}-06$ \\
hsa04914 & Progesterone-mediated oocyte maturation & 5 & $1.19 \mathrm{E}-05$ \\
hsa04010 & MAPK signaling pathway & 13 & $1.55 \mathrm{E}-05$ \\
hsa04510 & Focal adhesion & 11 & $2.80 \mathrm{E}-05$ \\
hsa04114 & Oocyte meiosis & 4 & $2.91 \mathrm{E}-05$ \\
hsa05212 & Pancreatic cancer & 7 & $3.11 \mathrm{E}-05$ \\
hsa05220 & Chronic myeloid leukemia & 7 & $4.03 \mathrm{E}-05$ \\
hsa04144 & Endocytosis & 3 & $4.63 \mathrm{E}-05$ \\
\hline
\end{tabular}

whose expressions were inversely correlated. Moreover, a global miRNA-target gene regulatory network was also constructed, in which several miRNAs and their targets were closely related to the development of HCC. Previous study indicated that miR-501-5p was significantly up-regulated in HCC and the over expression of miR-501-5p significantly promotes hepatitis B virus replication [28]. Analyzing the dataset of NCBI/GEO/GSE36915 also revealed that miR-501-5p was significantly up-regulated in HCC, and the over expression of miR-501-5p decreased CYLD expression which can promote the proliferation of HCC in vitro [29]. Consistent with that, we found that miR-501-5p was the most significantly upregulated miRNA in HCC compared with the normal tissue, and the validation result in the TCGA dataset was also in line with the integrated analysis. Our result provides additional evidence of the important role in the development of HCC and indicates that miR-501-5p will represent a powerful therapeutic target for HCC.

A recent study reported that miR-362-3p was up-regulated while methylation of its promoter significantly decreased in HCC compared with adjacent noncancerous tissues, and they suggested that deregulation of miR-362-3p and Tob2 may contribute to HCC malignancy [30]. Moreover, recent data revealed a clear correlation between HCC and aberrant MAPK8 in the pathogenesis of HCC, including gene expres- 
sion and prognostic outcomes. Therefore, targeting MAPK8 has been viewed as a new avenue for HCC therapy [31]. In the present study, miR-362-3p was up-regulated in HCC of both GEO and TCGA datasets. In addition, its target MAPK8 was down-regulated in this study, which confirmed that aberrant activation of protein kinases may play a causative role in the initiation and progression of the human HCC.

Additionally, it was reported that miR-769-3p can potentially interact in the 3' UTR region of PNPLA3, which was involved in the genetic susceptibility of nonalcoholic fatty liver disease in humans [32]. Herein, we found that miR-769-3p was significantly up-regulated, and the electronic validation result in the TCGA dataset was consistent with the integrated analysis. This suggested that it may be a potential novel therapeutic target for HCC.

Recent evidence showed that miR-941 was significantly down-regulated and generally hypermethylation in HCC. Moreover, the over expression of miR-941 suppressed in vitro cell proliferation, migration, and invasion and inhibited the metastasis of HCC cells in vivo [33]. However, we demonstrated that miR-941 was up-regulated in HCC compared with normal tissue, implying that the role of miR-941 in the progression of HCC needs to be further confirmed.

It was reported that loss of miR-16-1 was involved in the dedifferentiation and in the induction of chromosomal instability in dedifferentiated HCC with the extremely aggressive clinical behavior [34]. GLUD1 was a target of miR-16-1. Using a proteomics approach, Fillis found that maternal smoking can affect the expression of GLUD1 protein with a role in homeostasis of human fetal liver [35]. Herein, we found that miR-16-1 was up-regulated and GLUD1 was down-regulated in HCC, implying their roles in the onset and progression of HCC.

Previous study showed that miR-581 was down-regulated in in the tumor of HCC [36], and the down-regulation of miR-581 can promote hepatitis B virus surface antigen ( $\mathrm{Hb}$ sAg) expression by targeting Dicer and EDEM1, leading to a reduction in HBsAg expression and impede HCC development [37]. As the target of miR-581, SRPK2 was reported that it was closely related to the alternative splicing of Numb gene in HCC, which may play a role in the tumorigenesis $[38,39]$. Interestingly, the results of integrated analysis also indicated that miR-581 was down-regulated and SRPK2 was up-regulated, which revealed their expression pattern and regulation relative.

Besides, our integrated analysis also identified two novel differentially expressed miRNAs (miR-507 and miR-641), which obviously regulated the most target genes. Additionally, we also found one up-regulated miRNA (miR-502-3p) and three down-regulated miRNAs (miR-576-3p, miR-641 and miR-651) in HCC of GEO and TCGA datasets. Therefore, we speculated they may also play critical roles in the tumorigenesis of HCC. Their functions in HCC need to be further explored.

Additionally, functional annotation of differentially expressed target genes of differentially expressed miRNAs showed that these genes were significant enriched in the GO term for biological processes of toll signaling pathway, toll-like receptor 1 signaling pathway, toll-like receptor 2 signaling pathway and toll-like receptor 4 signaling pathway. Toll-like receptors (TLRs) are transmembrane signaling receptors that are widely expressed on parenchymal and non-parenchymal cells in the liver, which plays a major role in the liver health [40]. TLR1 and TLR2 can perform a heterodimer form that recognizes and activates various intracellular transcription factors and signaling molecules, which plays a crucial role in HCC [41]. Moreover, the TLR2-mediated immune network also plays an integrated defense role against HCC [42]. Additionally, it is stated TLR4 signaling plays part in hepatocellular tumorigenesis and progression $[43,44]$. Our result also showed that these target genes were remarkably enriched in toll signaling pathway in HCC, which was in line with the previous reports. This suggested the crucial role of toll signaling pathway in the development of HCC.

It is a known fact that miRNAs can act in concert to exert effects on target genes. Focusing on the interaction between miRNAs and target genes is likely to realistically capture potential pathology mechanism in HCC. In this study, the miRNA functional synergistic network, constructed by all miRNA synergistic pairs, revealed 17 differentially expressed miRNAs identified between HCC and control (6 up-regulated and 11 down-regulated). In this miRNA-mRNA regulatory network, miR-501-5p, miR-362-3p, miR-769-3p, miR-941, miR-16-1, miR-581, miR-507 and miR-641 and their target genes played an important role in the process of HCC. In a word, the network of miRNA-mRNA interactions indicated the complex regulatory mechanism of HCC and will help to improve understanding the regulatory mechanism of HCC and provide for developing novel therapeutic approaches.

\section{References}

[1] JEMAL A, CENTER MM, DESANTIS C, WARD EM. Global patterns of cancer incidence and mortality rates and trends. Cancer Epidemiol Biomarkers Prev 2010; 19: 1893-1907. https://doi.org/10.1158/1055-9965.EPI-10-0437

[2] NEGRINI M, FERRACIN M, SABBIONI S, CROCE CM. MicroRNAs in human cancer: from research to therapy. J Cell Sci 2007; 120: 1833-1840. https://doi.org/10.1242/jcs.03450

[3] Gailhouste L, Ochiya T. Cancer-related microRNAs and their role as tumor suppressors and oncogenes in hepatocellular carcinoma. Histol Histopathol 2013; 28: 437-451.

[4] MURAKAMI Y, YASUDA T, SAIGO K, URASHIMA T, TOYODA $\mathrm{H}$ et al. Comprehensive analysis of microRNA expression patterns in hepatocellular carcinoma and nontumorous tissues. Oncogene 2006; 25: 2537-2545. https:// doi.org/10.1038/sj.onc. 1209283

[5] LADEIRO Y, COUCHY G, BALABAUD C, BIOULAC-SAGE $\mathrm{P}$, PELLETIER L et al. MicroRNA profiling in hepatocellular tumors is associated with clinical features and oncogene/ tumor suppressor gene mutations. Hepatology 2008; 47: 1955-1963. https://doi.org/10.1002/hep.22256 
[6] GRAMANTIERI L, FORNARI F, CALLEGARI E, SABBIONI S, LANZA G et al. MicroRNA involvement in hepatocellular carcinoma. J Cell Mol Med 2008; 12: 2189-2204. https://doi. org/10.1111/j.1582-4934.2008.00533.x

[7] ARAVALLI RN, CRESSMAN EN, STEER CJ. Cellular and molecular mechanisms of hepatocellular carcinoma: an update. Arch Toxicol 2013; 87: 227-247. https://doi.org/10.1007/ s00204-012-0931-2

[8] KAN Z, ZHENG H, LIU X, LI S, BARBER TD et al. Wholegenome sequencing identifies recurrent mutations in hepatocellular carcinoma. Genome Res 2013; 23: 1422-1433. https://doi.org/10.1101/gr.154492.113

[9] VILLANUEVA A, LLOVET JM. Targeted therapies for hepatocellular carcinoma. Gastroenterology 2011; 140: 1410-1426. https://doi.org/10.1053/j.gastro.2011.03.006

[10] PINEAU P, VOLINIA S, MCJUNKIN K, MARCHIO A, BATTISTON C et al. miR-221 overexpression contributes to liver tumorigenesis. Proc Natl Acad Sci U S A 2010; 107: 264-269. https://doi.org/10.1073/pnas.0907904107

[11] JI J, SHI J, BUDHU A, YU Z, FORGUES M et al. MicroRNA expression, survival, and response to interferon in liver cancer. N Engl J Med 2009; 361: 1437-1447. https://doi.org/10.1056/ NEJMoa0901282

[12] FURUTA M, KOZAKI KI, TANAKA S, ARII S, IMOTO I et al. miR-124 and miR-203 are epigenetically silenced tumor-suppressive microRNAs in hepatocellular carcinoma. Carcinogenesis 2010; 31: 766-776. https://doi.org/10.1093/ carcin/bgp250

[13] LUPINI L, BASSI C, FERRACIN M, BARTONICEK N, D'ABUNDO L et al. miR-221 affects multiple cancer pathways by modulating the level of hundreds messenger RNAs. Front Genet 2013; 4: 64. https://doi.org/10.3389/ fgene.2013.00064

[14] TOME M, LOPEZ-ROMERO P, ALBO C, SEPULVEDA JC, FERNANDEZ-GUTIERREZ B et al. miR-335 orchestrates cell proliferation, migration and differentiation in human mesenchymal stem cells. Cell Death Differ 2011; 18: 985-995. https://doi.org/10.1038/cdd.2010.167

[15] VERONESE A, LUPINI L, CONSIGLIO J, VISONE R, FERRACIN $M$ et al. Oncogenic role of miR-483-3p at the IGF2/483 locus. Cancer Res 2010; 70: 3140-3149. https://doi. org/10.1158/0008-5472.CAN-09-4456

[16] GRAMANTIERI L, FERRACIN M, FORNARI F, VERONESE A, SABBIONI S et al. Cyclin G1 is a target of miR-122a, a microRNA frequently down-regulated in human hepatocellular carcinoma. Cancer Res 2007; 67: 6092-6099. https://doi. org/10.1158/0008-5472.CAN-06-4607

[17] SHIMIZU S, TAKEHARA T, HIKITA H, KODAMA T, MIYAGI $\mathrm{T}$ et al. The let-7 family of microRNAs inhibits Bcl$\mathrm{xL}$ expression and potentiates sorafenib-induced apoptosis in human hepatocellular carcinoma. J Hepatol 2010; 52: 698-704. https://doi.org/10.1016/j.jhep.2009.12.024

[18] MENG F, HENSON R, WEHBE-JANEK H, GHOSHAL $\mathrm{K}$, JACOB ST et al. MicroRNA-21 regulates expression of the PTEN tumor suppressor gene in human hepatocellular cancer. Gastroenterology 2007; 133: 647-658. https://doi. org/10.1053/j.gastro.2007.05.022
[19] BRACONI C, HUANG N, PATEL T. MicroRNA-dependent regulation of DNA methyltransferase-1 and tumor suppressor gene expression by interleukin-6 in human malignant cholangiocytes. Hepatology 2010; 51: 881-890. https://doi. org/10.1002/hep.23381

[20] SU H, YANG JR, XU T, HUANG J, XU L et al. MicroRNA-101, down-regulated in hepatocellular carcinoma, promotes apoptosis and suppresses tumorigenicity. Cancer Res 2009; 69: 1135-1142. https://doi.org/10.1158/0008-5472. CAN-08-2886

[21] CALLEGARI E, GRAMANTIERI L, DOMENICALI M, D'ABUNDO L, SABBIONI S et al. MicroRNAs in liver cancer: a model for investigating pathogenesis and novel therapeutic approaches. Cell Death Differ 2015; 22: 46-57. https://doi. org/10.1038/cdd.2014.136

[22] BARRETT T, WILHITE SE, LEDOUX P, EVANGELISTA C, KIM IF et al. NCBI GEO: archive for functional genomics data sets-update. Nucleic Acids Res 2013; 41: D991-995. https:// doi.org/10.1093/nar/gks1193

[23] MAROT G, FOULLEY JL, MAYER CD, JAFFREZIC F. Moderated effect size and P-value combinations for microarray meta-analyses. Bioinformatics 2009; 25: 2692-2699. https:// doi.org/10.1093/bioinformatics/btp444

[24] Tabas-Madrid D, Nogales-Cadenas R, Pascual-Montano A. GeneCodis3: a non-redundant and modular enrichment analysis tool for functional genomics. Nucleic Acids Res 2012; 40: W478-483. https://doi.org/10.1093/nar/gks402

[25] ASHBURNER M, BALL CA, BLAKE JA, BOTSTEIN D, BUTLER $\mathrm{H}$ et al. Gene ontology: tool for the unification of biology. The Gene Ontology Consortium. Nat Genet 2000; 25: 25-29. https://doi.org/10.1038/75556

[26] KANEHISA M, GOTO S. KEGG: kyoto encyclopedia of genes and genomes. Nucleic Acids Res 2000; 28: 27-30. https://doi. org/10.1093/nar/28.1.27

[27] XUE J, NIU J, WU J, WU ZH. MicroRNAs in cancer therapeutic response: Friend and foe. World J Clin Oncol 2014; 5: 730-743. https://doi.org/10.5306/wjco.v5.i4.730

[28] JIN J, TANG S, XIA L, DU R, XIE H et al. MicroRNA-501 promotes HBV replication by targeting HBXIP. Biochem Biophys Res Commun 2013; 430: 1228-1233. https://doi. org/10.1016/j.bbrc.2012.12.071

[29] HUANG DH, WANG GY, ZHANG JW, LIi Y, ZENG XC et al. MiR-501-5p regulates CYLD expression and promotes cell proliferation in human hepatocellular carcinoma. Jpn J Clin Oncol 2015; 45: 738-744. https://doi.org/10.1093/jjco/hyv063

[30] SHEN H, LI W, TIAN Y, XU P, WANG H et al. Upregulation of miR-362-3p Modulates Proliferation and Anchorage-Independent Growth by Directly Targeting Tob2 in Hepatocellular Carcinoma. J Cell Biochem 2015; 116: 1563-1573. https://doi. org/10.1002/jcb.25110

[31] CHEN F, BEEZHOLD K, CASTRANOVA V. JNK1, a potential therapeutic target for hepatocellular carcinoma. Biochim Biophys Acta 2009; 1796: 242-251. https://doi.org/10.1016/j. bbcan.2009.06.005

[32] SOOKOIAN S, PIROLA CJ. PNPLA3, the triacylglycerol synthesis/hydrolysis/storage dilemma, and nonalcoholic fatty liver disease. World journal of gastroenterology World J 
Gastroenterol 2012; 18: 6018-6026. https://doi.org/10.3748/ wig.v18.i42.6018

[33] ZHANG PP, WANG XL, ZHAO W, QI B, YANG Q et al. DNA methylation-mediated repression of miR-941 enhances lysine (K)-specific demethylase 6B expression in hepatoma cells. J Biol Chem 2014; 289: 24724-24735. https://doi.org/10.1074/ jbc.M114.567818

[34] SKAWRAN B, STEINEMANN D, BECKER T, BUURMAN R, FLIK J, et al. Loss of $13 q$ is associated with genes involved in cell cycle and proliferation in dedifferentiated hepatocellular carcinoma. Modern pathology 2008; 21: 1479-1489. https:// doi.org/10.1038/modpathol.2008.147

[35] FILIS P, NAGRATH N, FRASER M, HAY DC, IREDALE JP et al. Maternal Smoking Dysregulates Protein Expression in Second Trimester Human Fetal Livers in a Sex-Specific Manner. J Clin Endocrinol Metab 2015; 100: E861-870. https:// doi.org/10.1210/jc.2014-3941

[36] KATAYAMA Y, MAEDA M, MIYAGUCHI K, NEMOTO $S$, YASEN $M$ et al. Identification of pathogenesis-related microRNAs in hepatocellular carcinoma by expression profiling. Oncol Lett 2012; 4: 817-823.

[37] WANG YQ, REN YF, SONG YJ, XUE YF, ZHANG XJ et al. MicroRNA-581 promotes hepatitis B virus surface antigen expression by targeting Dicer and EDEM1. Carcinogenesis 2014; 35: 2127-2133. https://doi.org/10.1093/carcin/bgu128

[38] WANG Z, LI SS. Numb: A new player in EMT. Cell Adh Migr 2010; 4: 176-179. https://doi.org/10.4161/cam.4.2.10690
[39] LU Y, XU W, JI J, FENG D, SOURBIER C et al. Alternative splicing of the cell fate determinant Numb in hepatocellular carcinoma. Hepatology 2015; 62: 1122-1131. https://doi. org/10.1002/hep. 27923

[40] KUMAR H, KAWAI T, AKIRA S. Toll-like receptors and innate immunity. Biochem Biophys Res Commun 2009; 388: 621-625. https://doi.org/10.1016/j.bbrc.2009.08.062

[41] LIANG Z, WU S, LI Y, HE L, WU M et al. Activation of Tolllike receptor 3 impairs the dengue virus serotype 2 replication through induction of IFN-beta in cultured hepatoma cells. PLoS One 2011; 6: e23346. https://doi.org/10.1371/journal. pone.0023346

[42] LIN H, YAN J, WANG Z, HUA F, YU J et al. Loss of immunity-supported senescence enhances susceptibility to hepatocellular carcinogenesis and progression in Toll-like receptor 2-deficient mice. Hepatology 2013; 57: 171-182. https://doi.org/10.1002/hep.25991

[43] WANG Z, YAN J, LIN H, HUA F, Wang X et al. Toll-like receptor 4 activity protects against hepatocellular tumorigenesis and progression by regulating expression of DNA repair protein Ku70 in mice. Hepatology 2013; 57: 1869-1881. https://doi. org/10.1002/hep.26234

[44] WANG L, ZHU R, HUANG Z, LI H, ZHU H. Lipopolysaccharide-induced toll-like receptor 4 signaling in cancer cells promotes cell survival and proliferation in hepatocellular carcinoma. Dig Dis Sci 2013; 58: 2223-2236. https://doi. org/10.1007/s10620-013-2745-3 


\section{Supplementary Table S1}

\begin{tabular}{lrrrrrrrr} 
id & expCase & expCtrl & rawpvalp & fdrp & \multicolumn{2}{c}{ rawpvalES } & fdrES & updown \\
hsa-miR-933 & $-0,801877$ & $-0,41342$ & $1,772 E-11$ & $4,615 E-09$ & $1,542 E-08$ & $3,59 E-06$ & -1 down \\
hsa-miR-501-5p & 1,0007853 & 0,4362344 & $7,973 E-09$ & $7,547 E-07$ & $2,528 E-08$ & $3,59 E-06$ & 1 up \\
hsa-miR-769-3p & 0,1318229 & $-0,270927$ & $5,071 E-08$ & $2,086 E-06$ & $1,095 E-07$ & $1,037 E-05$ & 1 up \\
hsa-miR-651 & 0,6315291 & 1,0853643 & $2,78 E-07$ & $7,178 E-06$ & $5,29 E-07$ & $3,063 E-05$ & -1 down \\
hsa-miR-924 & $-0,187079$ & 0,2500933 & $3,278 E-07$ & $7,758 E-06$ & $5,393 E-07$ & $3,063 E-05$ & -1 down \\
hsa-miR-502-5p & 0,3459062 & 0,6961232 & $4,296 E-07$ & $9,385 E-06$ & $7,451 E-07$ & $3,527 E-05$ & -1 down \\
hsa-miR-941 & 0,5556343 & 0,0442101 & $1,139 E-06$ & $1,903 E-05$ & $1,772 E-06$ & $7,189 E-05$ & 1 up \\
hsa-miR-362-3p & 1,2509277 & 0,6976929 & $1,382 E-06$ & $2,181 E-05$ & $2,171 E-06$ & $7,706 E-05$ & 1 up \\
hsa-miR-641 & 0,0984104 & 0,304479 & $2,131 E-06$ & $3,026 E-05$ & $3,222 E-06$ & 0,0001017 & -1 down \\
hsa-miR-597 & $-0,645011$ & $-0,463781$ & $3,082 E-05$ & 0,0003018 & $4,538 E-05$ & 0,0012889 & -1 down \\
hsa-miR-581 & 0,0338183 & 0,3237183 & 0,0001673 & 0,0013574 & 0,0002347 & 0,0060588 & -1 down \\
hsa-miR-576-3p & $-0,110769$ & 0,1411198 & 0,0002729 & 0,001987 & 0,0003664 & 0,0086708 & -1 down \\
hsa-miR-507 & 0,4223288 & 0,7500609 & 0,0003518 & 0,0024978 & 0,0004357 & 0,0095183 & -1 down \\
hsa-miR-502-3p & 1,4311922 & 1,0402696 & 0,0003807 & 0,0026367 & 0,00052 & 0,0097541 & 1 up \\
hsa-miR-16-1* & 1,3995254 & 1,0051008 & $9,005 E-07$ & $1,705 E-05$ & 0,0005699 & 0,0097541 & 1 up \\
hsa-miR-569 & $-0,74605$ & $-0,616621$ & 0,0004286 & 0,0028549 & 0,0005799 & 0,0097541 & -1 down \\
hsa-miR-518d-3p & 0,418345 & 0,6019967 & 0,0004323 & 0,0028549 & 0,0005839 & 0,0097541 & -1 down
\end{tabular}

